\title{
MODELING OF PULSAR POLARIZATION PROPERTIES
}

\author{
L. M. SHIER \\ Physics Department, Rice University \\ Sponsored by \\ F. C. MICHEL \\ Space Physics and Astronomy Department, Rice University
}

\begin{abstract}
In the popularly accepted empirical model for pulsar emission, bunches of charged particles traveling along open field lines near the magnetic pole emit curvature radiation. Such radiation is linearly polarized. Most of the radiation is assumed to be emitted from a ring shaped region centered on the pole (the hollow cone model). We have calculated the expected average polarization using this model and find them to be in disagreement with observations. The addition of a second ring inside the first with orthogonal polarization solves this problem. This new model explains other observed features of pulsar emission including discontinuities in position-angle profiles and multicomponent profiles. Plasma interactions might account for a second ring.
\end{abstract}

\section{Introduction ${ }^{1}$}

This was a senior physics research project in radio pulsars. The natural sort of project for a student having little theoretical background (especially in plasma physics, etc.) was one in pulsar pulse morphology. I had recently suggested that orthogonal mode changes might originate from the overlap of fan beams from distinct localized sources (Michel $1987 \mathrm{~b}$ ), and in the back of my mind I thought this would be an opportunity to check out the statistics of that idea. Moreover, Lyne and Manchester (1988) had just come out with an extensive tabulation and analysis of pulsar profiles, which provided one of a number of valuable data bases from which to work.

The project evolved through a number of stages, not so much because any one stage was unprofitable, but rather because each led to a more interesting one. Originally the idea was to model pulsar emission according to the hollow cone model with emission from discrete sources dotted along the cone, each emitting $100 \%$ polarized radiation parallel to the local magnetic field angle, with a Gaussian width and a Gaussian extent. Most invocations of the hollow cone model are qualitative, and the few quantitative applications usually deal with positionangle swings. Once the program for this ring model was working, the intensity, polarization, and position angles were calculated for various observing angles and inclination angles, with results reminiscent of actual pulsar profiles and polarization swings. A fundamental problem became clear early on: there

\footnotetext{
${ }^{1}$ The introduction is by $F$. C. Michel. The remainder of the paper is by L. M. Schier
}

are a lot of free parameters ( 5 just to convert the idea of hollow cone emission into the ring model). A fit to any given pulsar would not necessarily prove much of anything. So we started out to do statistics, based on the premise that the observer's position is not special. The proportion of singles to doubles (this ring model would not give triples, of course) should be about the same for other observers in the galaxy as for us. We could therefore take a given model, view it from all angles, and classify the resultant pulse profiles. The next step would have been to iterate the model parameters to mimic the observed ones, address the issue of triples, and worry about a host of detaikss. But the ring model failed to correctly give the observed polarization properties of the doubles!

The source of the failure, as is so often the case, can be seen without recourse to numerical modeling. A strongly polarized pulsar like PSR 0833-45 is nearly $100 \%$ polarized, which also strongly suggests that the basic underlying emission mechanism is similarly polarized, hence the ring model. Yet if one simply picks at random a pulsar having two or three components, the polarization rarely exceeds $50 \%$. A ring model will only give depolarization because the two pulses (when ring is clipped twice by the line of sight) overlap. Low polarization is mutually exclusive of cleanly separated double pulses; if the pulses are cleanly separated, there is not enough overlap to give anything like $50 \%$ depolarization. There seemed no point in trying to do statistics with a model that could not match the observed pulses properties.

At this point, we had a model restricted to single and double profiles that could not correctly give the polarization of the latter, much less give triples. 
Reducing the ring emission to two or three spots did not seem to help (despite this being on my original agenda). Shortly thereafter, Lisa Shier walked into my office and said that a double ring, with the polarization from the one orthogonal to the polarization from the other, worked better. Indeed it did. One also got triples and orthogonal mode changes! And for the most part the depolarization (in the model) simply results from having two overlapping (for the most part) sources in the same line of sight.

At this point we could have returned to try statistics, but the end of the course had come. What follows is the report of the work, slightly edited because it was directed at a different audience. I should also note that figures 3 and 4 were not in any way optimized to try to reproduce all of the features of the two pulsars in question.

\section{The hollow cone model}

One of the most popular models, especially among observers, is the hollow cone model. The proposed emission mechanism is curvature radiation. Bunches of charged particles travel along the open field lines. Since the field lines are curved, the particles are accelerated. The radiation that they emit is linearly polarized in the plane of curvature of the field line. Curvature radiation is very directional. It is strongest in the direction of the particle's instantaneous velocity and falls off as a known function of angle from that axis. Most of the radiation occurs near the edges of the open field line region because the field lines are more sharply curved here. In an approximately dipolar field, the radiation will be emitted perpendicular to the surface. The locus of points from which the observer will see radiation is a line of constant latitude, a parallel. As the pulsar turns, the origin of radiation seen by the observer will change.

For an individual pulse, there may not be emission on a given field line. However, the average intensity of any spot on the pulsar surface is a function only of distance to the magnetic pole. Calculations using this average intensity will give average pulse shapes.

As it stands, this model is capable of describing qualitatively what the total intensity profiles should look like and giving a quantitative description of the position-angle curves. If the line of sight passes near the magnetic pole a two-component profile will be seen. A larger closest approach to the pole will give the observer a one-component profile. It is also possible that the pulsar remains unseen. If the emitting region is regarded as almost flat and a polar coordinate system is set up on it such that a ray with $\phi=0$ goes through the north pole (geographic) then the position angle for any given spot is $\phi+c$. Since the line of sight is approximately $b=r \tan \phi$, the position angle will be given by $\arctan (b / r)+c$. This relationship is a good description of many of the observed position angle curves (Manchester and Taylor 1977).

The model has three free parameters. The size of the emitting region will affect how much the position-angle changes over the course of a pulse. The pulse duration implies that the angular radius for this region is between $4^{\circ}$ and $8^{\circ}$. The distance of closest approach to the magnetic pole determines the pulse shape and also the position-angle swing. This is probably random; there is no reason to assume a correlation between the orientation of the pulsar's rotational axis and the direction of Earth. The latitude of the pulsar's magnetic poles determines the rate at which the point emitting toward Earth changes, and hence what percentage of the pulse the pulsar is visible.

\section{The ring model}

More assumptions must be made in order to get a model which can be used to calculate the values of the total intensity and the polarized intensity. First, intensity as a function of the distance from the magnetic pole is required. The ring model assumes that all of the emission is from a ring at the edge of the open field line region. The field lines are mosi strongly curved there, so emission from this area will be more intense than from elsewhere. In order to avoid numerical computation of complicated integrals, the ring is modeled as a large number of discrete spots. The emission from each spot is added incoherently to get the total emission. In essence, an integral is being replaced by a sum.

For a large number of points on the sight-line path, the emission from each spot in the direction of the normal at that point is calculated. In a direction perpendicular to a spot's polarization axis, the radiation is assumed to fall off like curvature radiation. This function is described by a characteristic width parameter. In the direction parallel to the polarization direction (the radial direction) the intensity is assumed to fall off as a Gaussian. The Gaussian distribution will model what happens if radiation is emitted from more than one location on a field line or from particles on a number of field lines.

The model has five free parameters: the latitude of the magnetic pole and the latitude of the sight line path are the same as in the hollow cone model. The ring radius is similar to the size of the emitting region for the hollow cone model, but it must be slightly smaller to fit the data since emission does 
not abruptly stop when the sight line point passes outside the ring. The characteristic widths of the intensity distribution are the last two.

\section{Results from the ring model}

The calculated curves from this model are similar to those of real pulsars in a number of ways. Both one and two-component profiles can be obtained. The position angles vary smoothly with longitude. A great deal of linear polarization is present.

However, there are a number of inconsistencies between pulsar data and the model's predictions. A number of three-component pulsars exist. No combination of parameters for this model will give a three-component profile. There are also pulsars which have $90^{\circ}$ discontinuities in their position-angle curves, instead of smooth, continuous position-angle curves.

Even more troubling is the observation that the amount of polarization is too high for pulsars whose intensity and position-angle curves are well described by the model. The amount of polarization of a pulsar is usually described by measuring the average energy of each pulse and the average energy of the polarized emission, and then taking a ratio to get the average polarization.

In the case of two-component profiles, we classified the degree of separation according to

$$
\frac{\text { Intensity at the central minimum }}{\text { Maximum intensity }}=\frac{I_{\mathrm{v}}}{I_{\mathrm{m}}}
$$

If $I_{\mathrm{v}} / I_{\mathrm{m}}$ is near 1 , the profile looks much like a one-component pulsar, whereas for lower values, the peaks in the total intensity curve become increasingly distinct. The minimum value of the average polarization that the model would allow was calculated as a function of $I_{\mathrm{v}} / I_{\mathrm{m}}$.

To compare this with real pulsars, four surveys of pulsars were found (Hamilton et al. 1977a, Manchester, Hamilton, and McCulloch 1980, McCulloch et al. 1978, Morris et al. 1981). A total of 22 two-component pulsars were included. Of these, several had been observed at two or more frequencies, for a total of 37 two-component profiles. The ratio $I_{\mathrm{v}} / I_{\mathrm{m}}$ was measured from plots of the profiles and catalogued together with the listed average polarization. This data was then compared with the model's predicted minimums as shown in figure 1. Only one pulsar fell above the minimum curve, and three above the curve's lowest value. This model does not work for two-component pulsars. Asymmetry in emission makes the polarization problems worse. If one uses emission from only two spots polarized $90^{\circ}$ apart, there is a minimum

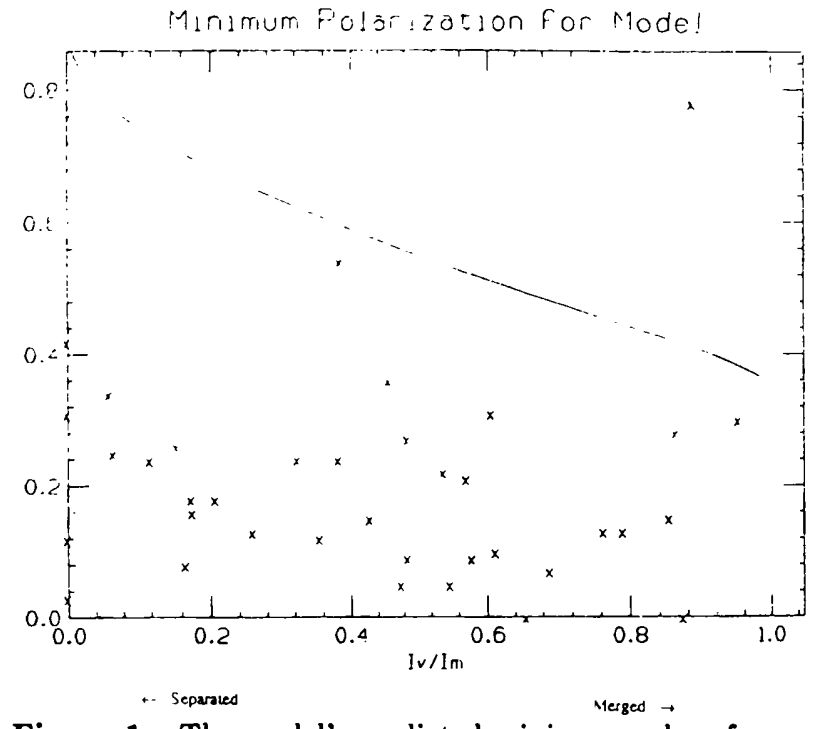

Figure 1 The model's predicted minimum values for average fraction of polarization as a function of the parameter $I_{\mathrm{v}} / I_{\mathrm{m}}$. The $x$ 's are measurements of actual pulsars. The model profiles go from well separated to merged as $I_{\mathrm{v}} / I_{\mathrm{m}}$ goes from 0 to 1 .

average polarization of $43 \%$ for pulsars with twocomponent profiles. This is higher than the $36 \%$ minimum of the symmetric case.

The case of single-component pulsars can also be considered. For a given single-component pulsar, the polarization can approach zero. However, this is not true of an ensemble of pulsars if the orientation of the rotational axis is random. If the sight line path does closely approach the magnetic pole, the pulsar has a high degree of polarization.

It is possible to use the model to predict the ensemble-average polarization. First, assume that the half width of the Gaussian part of the intensity distribution equals the radius of the ring emission. This will produce the lowest ensemble polarization of any reasonable parameter set. Weight the average polarization of each configuration by its maximum intensity, which is proportional to the number of such pulsars visible. This procedure yields an ensemble-average polarization of $40 \%$.

The surveys used for comparing two-component pulsars contained a total of $\mathbf{2 4 6}$ observations of pulsars. Eighty pulsars were observed at least once, and many of them were observed at more than one frequency. The ensemble average polarization for this collection of pulsars is $19 \%$. This model does not work for ensembles of pulsars either.

\section{The double-ring model}

The double-ring model uses the emission configuration of the ring model but adds a second ring inside the first which emits radiation polarized perpendicular to the field line as shown in figure 2. 

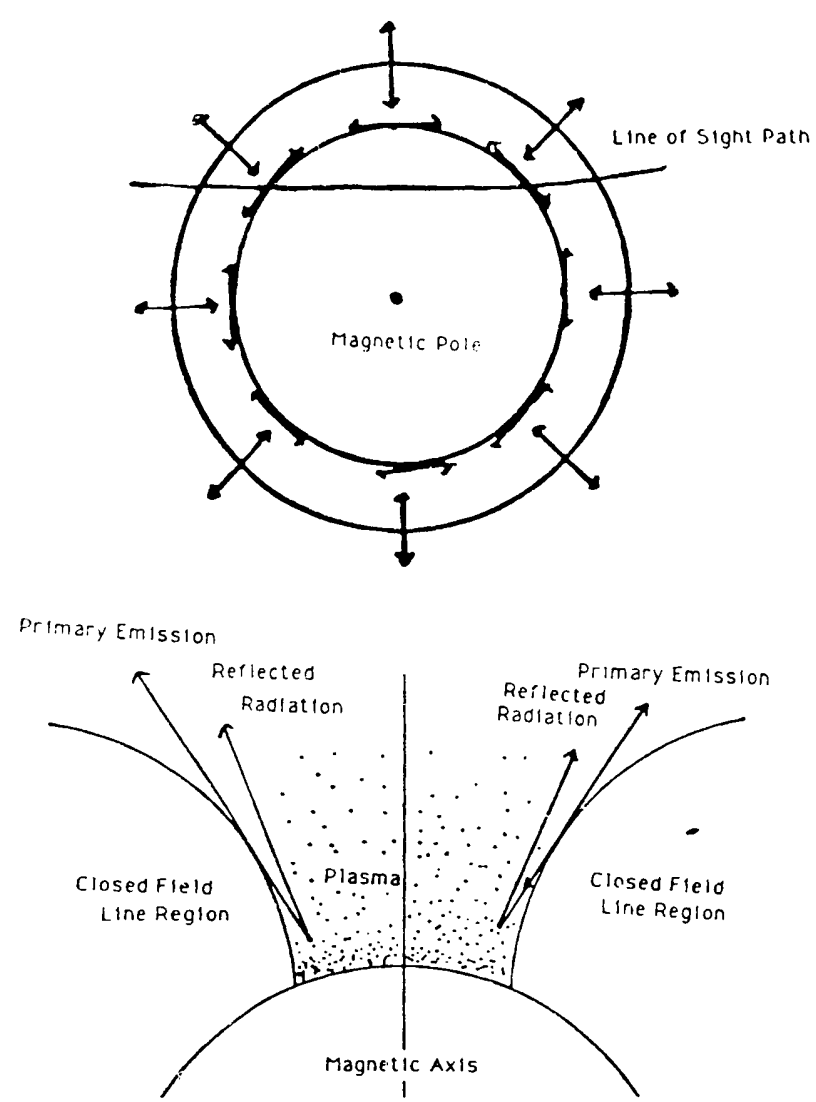

Figure 2 Geometry of emission for the double-ring model; arrows denote the polarization direction.

The proposed source for the second ring is reflection from the primary ring. Some particles will travel outward along the field lines and thus radiate outward. Others, with opposite charge, will radiate inward. This radiation can interact with the plasma that fills the polar cap region of the pulsar. When the number of charged particles reaches a critical density, the radiation will be reflected. The position-angle change of $90^{\circ}$ may have something to do with the two different propagation paths.

This model assumes that the radiation from a spot on either ring falls off as a Gaussian. This may not be entirely correct, but it reduces the number of free parameters to a reasonable level. In the single ring model, the curvature-radiation characteristic width parameter did not usually affect the calculated curves a great deal. The reflection processes may not be $100 \%$ efficient; the efficiency is another free parameter in the model. The others are the magnetic pole latitude, sight-line path latitude and the radii of the two rings.

\section{Results from the double-ring model}

Quantitative comparisons of this model with actual data have not been done, in fact, it is hard to know what is appropriate. However, many interesting features of this model can be observed in a qualitative way.

The polarization of the calculated pulsars is much lower than for the single-ring model. In the limit that the rings overlap, have the same intensity distributions, and reflection efficiency, $100 \%$ polarization goes to zero for any choice of the other parameters. This allows one to produce a profile with total intensity exactly like a ring-model ana$\log$, but with no polarization. Nonzero minimum polarization and minimum ensemble polarizations are impossible to compute without further restrictions on the parameters. It is not clear what these restrictions should be. Working out the details of the reflection mechanism may provide suitable constraints.

Calculated position-angle profiles may be smooth or contain two $90^{\circ}$ discontinuities. These discontinuities have been observed in a number of pulsars.

The double-ring model can produce pulsars with one to four components. Three-component pulsars are rare in nature but they have been observed. This model predicts that all such pulsars will have two $90^{\circ}$ discontinuities in the position-angle profile. There is some data to support this, but a few published profiles show a smooth position-angle profile (Lyne and Manchester 1988) as shown in figure 3. It is not clear whether there are three-component pulsars with smooth position-angle curves, or if this is an artifact of the data gathering process. A threecomponent profile necessarily has a great deal of structure. Low time resolution can cause that structure to be lost. This is particularly a problem with faint or fast pulsars.

One study looked at the distribution of positionangle measurements of individual pulses at the same part of the pulse. The authors found that their measurements often did not have a Gaussian distribution about a central value, but rather had two peaks $90^{\circ}$ apart. The authors hypothesized that there are two orthogonal sources, neither of which is emitting continuously. The source that was brightest at the time of an individual pulse would determine the position angle (Stinebring et al. 1984b). The current model adds a mechanism and an emission geometry to that hypothesis.

The double-ring model can accurately describe the profile shape of a real pulsar. An attempt was made to match the profiles of PSR 1039-19 at $408 \mathrm{MHz}$ (Lyne and Manchester 1988) as shown in figure 4. This pulsar has a rapid drop in intensity on the outer edges of the pulse and a slower decline towards the inner minimum. The model is able to match the shape of the peaks with reasonable accuracy. Also, the four peaks in the polarized intensity 
Observed Prollles

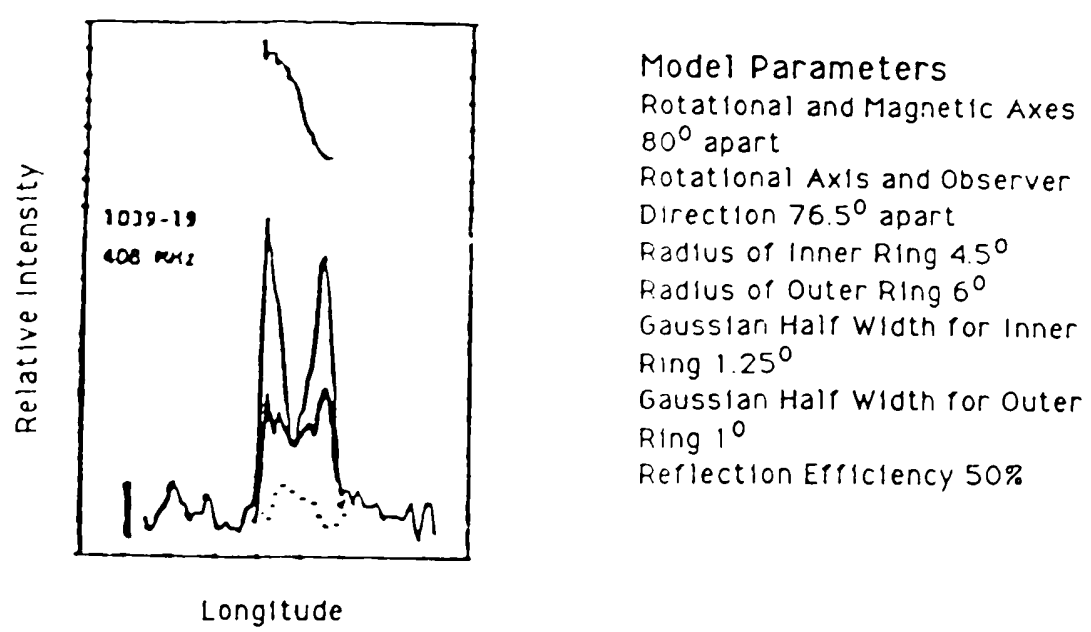

Model Proflles
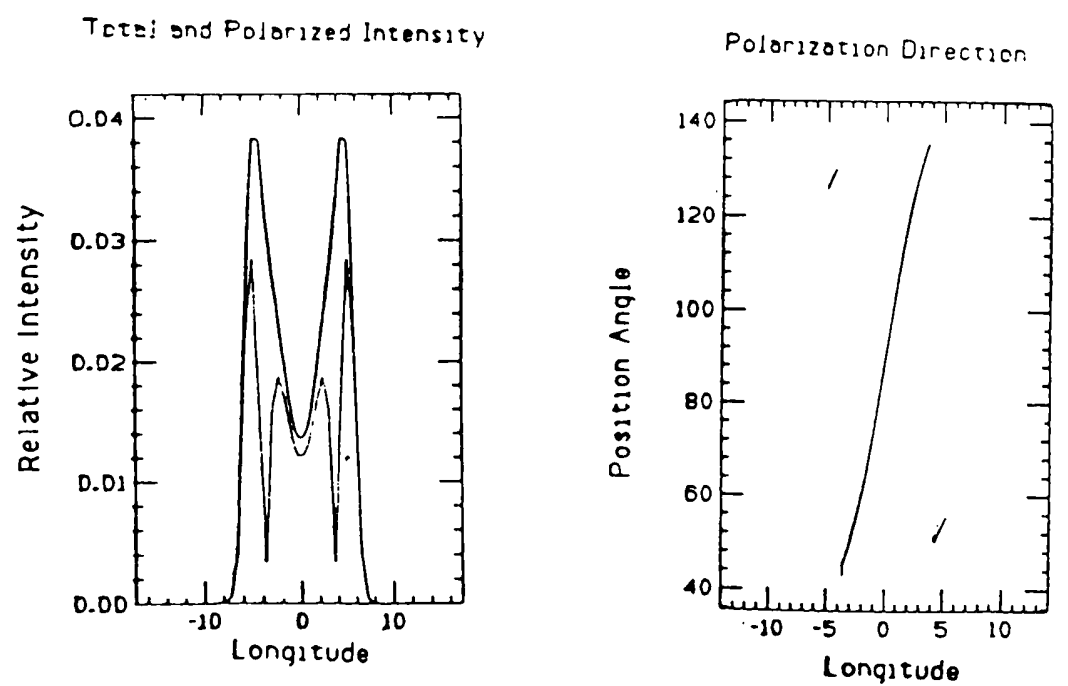

Figure 3 Measured average profile and a model fit.

curve are seen. The calculated position angle does have discontinuities but these may not have been observed because of time resolution problems.

\section{Asymmetry and circular polar- ization}

This project has focused on the total intensity and linear polarization. There are two other properties of the average profiles that deserve mention. Many pulsars have a small amount of circular polarization. In the current double-ring model, circular polarization is neglected. The model itself does not necessarily lead to zero circular polarization. Curvature radiation does have a circularly polarized component when observed off axis, as it is in this model. This is a detail that could be added to the model.

Pulsar profiles are usually rather symmetric, which leads to the development of models with symmetry. Perhaps the small asymmetries seen in most pulsars could be treated as a perturbation of the symmetric case. A somewhat nondipolar field would introduce some distortions. The ring might not be emitting uniformly. Some pulsars exist which look like "half" of a two-component pulsar.

\section{Conclusions}

The popular hollow cone model of emission is not a complete description of pulsar emission processes, because it lacks details on the intensities of different areas of the emitting region. This makes calculations of total intensity and polarized intensity impossible. The ring model, a version of the hollow cone model which does contain enough information to calculate profiles, is also unsuitable. 

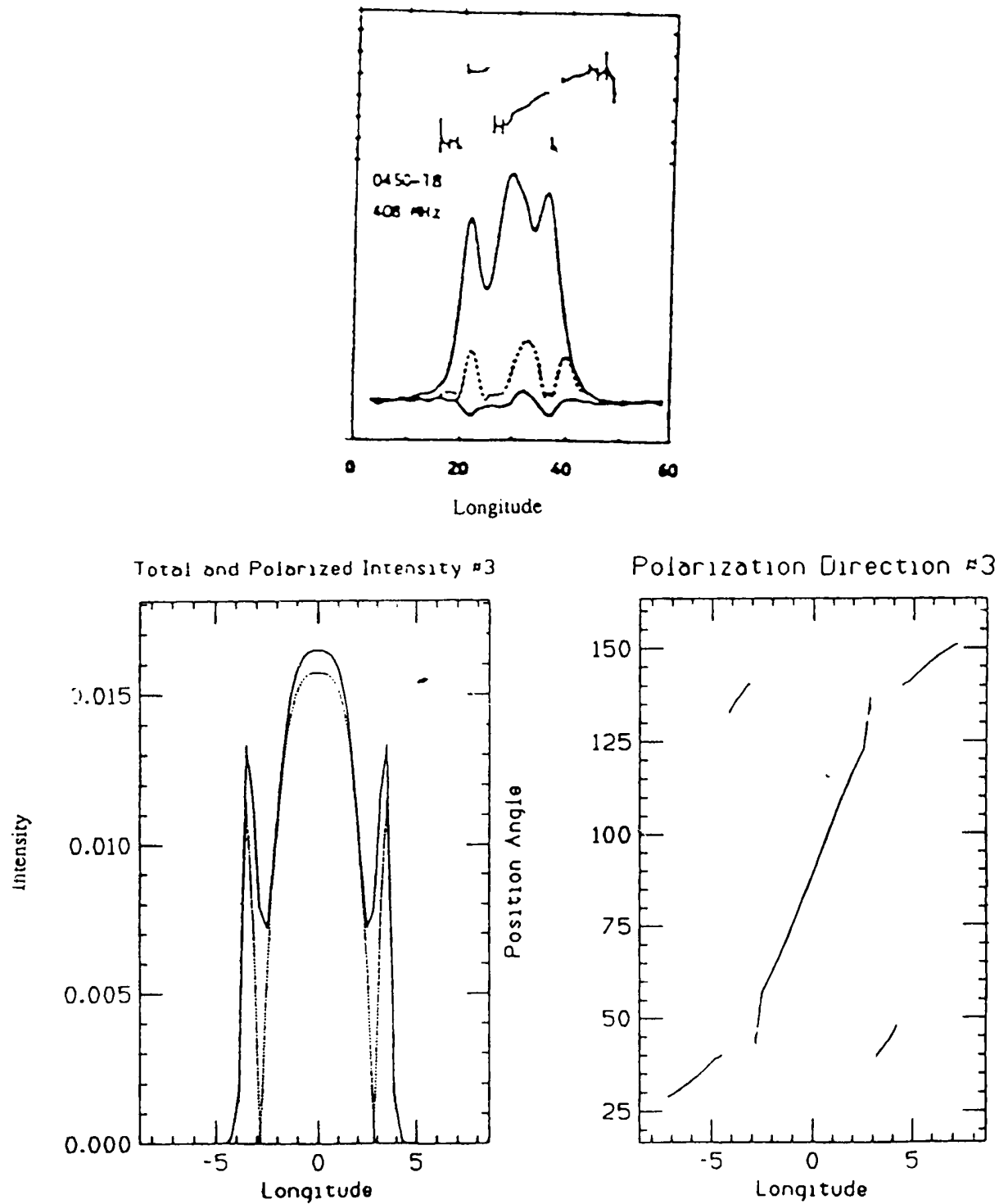

Figure $490^{\circ}$ discontinuitues.

There are problems with the total intensity, polarized intensity and position-angle profiles. A modification is suggested. The double-ring model more closely fits the observed data and seems to be qualitatively close to correct. Quantitative comparisons will be easier if the physics of whatever might cause doubling reflection is better understood. Observations with better time resolution will help to test the model. 